\title{
Editorial
}

\section{Machine Learning with Applications to Autonomous Systems}

\author{
Xin Xu, ${ }^{1}$ Haibo He, ${ }^{2}$ Dongbin Zhao, ${ }^{3}$ Shiliang Sun, ${ }^{4}$ Lucian Busoniu, ${ }^{5}$ and Simon X. Yang ${ }^{6}$ \\ ${ }^{1}$ College of Mechatronics and Automation, National University of Defense Technology, Changsha 410073, China \\ ${ }^{2}$ Department of Electrical, Computer, and Biomedical Engineering, University of Rhode Island, Kingston, RI 02881, USA \\ ${ }^{3}$ State Key Lab of Management and Control for Complex Systems, Institute of Automation, Chinese Academy of Sciences, \\ Beijing 100190, China \\ ${ }^{4}$ Department of Computer Science and Technology, East China Normal University, Shanghai 200241, China \\ ${ }^{5}$ Automation Department, Technical University of Cluj-Napoca, 400609 Cluj-Napoca, Romania \\ ${ }^{6}$ School of Engineering, University of Guelph, Guelph, ON, Canada N1G 2W1 \\ Correspondence should be addressed to Xin Xu; xinxu@nudt.edu.cn
}

Received 6 July 2015; Accepted 6 July 2015

Copyright (C) 2015 Xin Xu et al. This is an open access article distributed under the Creative Commons Attribution License, which permits unrestricted use, distribution, and reproduction in any medium, provided the original work is properly cited.

Autonomous systems are a class of intelligent systems that can realize autonomous sensing, modeling, decision-making, and control in uncertain, dynamic environments. Typical examples include autonomous vehicles and intelligent software agents in networks. In the past decade, theory and applications of autonomous systems have been investigated from multidisciplinary perspectives including machine learning, pattern recognition, robotics, and intelligent control. In particular, to realize adaptive sensing, modeling, planning, and control for autonomous systems, various machine learning methods play an important role in discovering knowledge from observed data. In this context, due to the increasing complexity of real-world applications, it is necessary for autonomous systems to have improved learning abilities such as online learning, learning from imbalanced data, for sensing, planning, and motion control. Efforts to address these difficulties include incremental learning from stream data, semisupervised learning, online reinforcement learning (RL), and approximate dynamic programming (ADP). However, there are still many challenges in developing efficient machine learning algorithms with good generalization ability for autonomous systems in uncertain, dynamic environments.

The aim of this special issue is to provide a forum for different research efforts towards new machine learning theory and algorithms with applications to autonomous systems. After a rigorous reviewing process, 9 papers were accepted among more than two dozens of submitted manuscripts. The topics of these 9 papers can be categorized into three groups.

The first group of papers focused on new machine learning algorithms for difficult learning tasks in autonomous sensing and modeling, such as learning from stream data, semisupervised learning, learning from imbalanced data, and so forth. In one of the papers, F. Cheng et al. proposed a structural SVM framework for binary classification problems under class imbalance. The structural SVM framework was designed to directly optimize the performance measures for imbalanced data. In another paper, by introducing a Memorizing-based Data Stream Mining (MDSM) model, Y. Jiang et al. presented a stream data learning algorithm called MAE (Memorizing based Adaptive Ensemble), where a forgetting strategy is designed based on a forgetting curve and a recalling strategy is given based on ensemble pruning. The paper by Y. Zhou and S. Sun focused on semisupervised learning methods for dimension reduction, where a new regularizer based on tangent spaces is proposed to preserve the local manifold property in a semisupervised way. The proposed method is called Semisupervised Tangent Space Discriminant analysis and the global optimal solution can be obtained by solving a generalized eigenvalue problem.

The second group of papers investigated a collection of autonomous sensing problems and proposed specific learning-based or data-driven feature extraction and classification methods for these problems. By combining 
the local Gabor features and a spatial pyramid matching model, B. Dong and G. Ren proposed a new scene classification approach with a modified kernel principal component analysis algorithm. In the paper by S. Zhu and L. Xia, the human action recognition problem in video data was studied, where silhouette features were combined with local optical flow features and an improved multi-instance learning algorithm was used. After introducing the concept of microcommunity, J. Qi et al. proposed a community clustering algorithm for community mining in complex networks. $\mathrm{X}$. Zhang et al. presented a Delaunay-based stereo matching method which aims to compute the disparity map with reduced computational costs. In this method, the selection of the parameters was realized in a data-driven way, whereas some learning-based methods may be developed to improve the performance further.

The third and final group of papers comprises two works that are dedicated to solve learning-based control problems. Aiming at reinforcement learning methods for autonomous control systems, $\mathrm{X}$. Chen et al. presented a twostage simultaneous learning method for a policy evaluation network using Gaussian kernels. Then, by combining the critic learning method in an actor-critic learning control framework, a Gaussian-kernel-based adaptive dynamic programming (GKADP) approach was also developed. In the last paper of this issue, M. AlDhaifallah applied twin support vector machines to model identification of nonlinear Wiener systems. Two simulation examples were provided to show the performance of twin support vector regression in identifying nonlinear Wiener models.

In order to deal with the upcoming challenges in autonomous systems, it is necessary to develop more advanced machine learning techniques and theories in the literature. We hope that the collected papers in this special issue can shed some light on future innovative work.

\section{Acknowledgments}

As the guest editors of the special issue, we would like to thank all the authors and reviewers for their contributions to our special issue.

$X i n X u$

Haibo $\mathrm{He}$

Dongbin Zhao Shiliang Sun

Lucian Busoniu

Simon X. Yang 


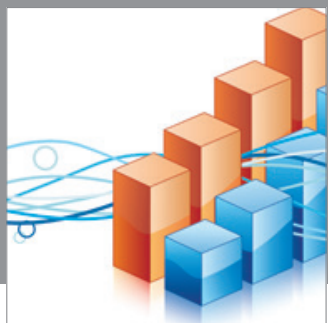

Advances in

Operations Research

mansans

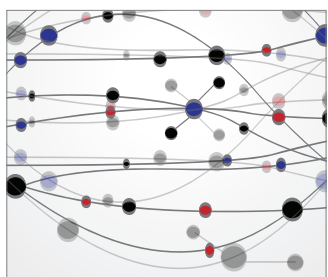

The Scientific World Journal
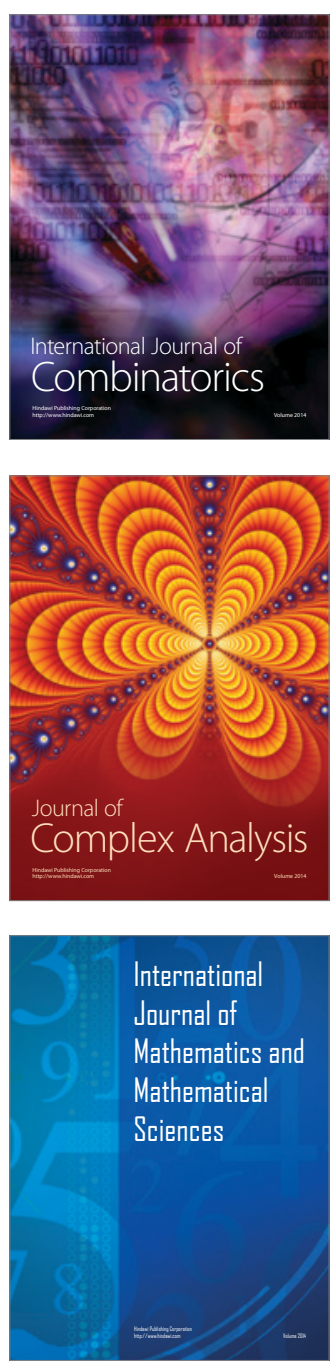
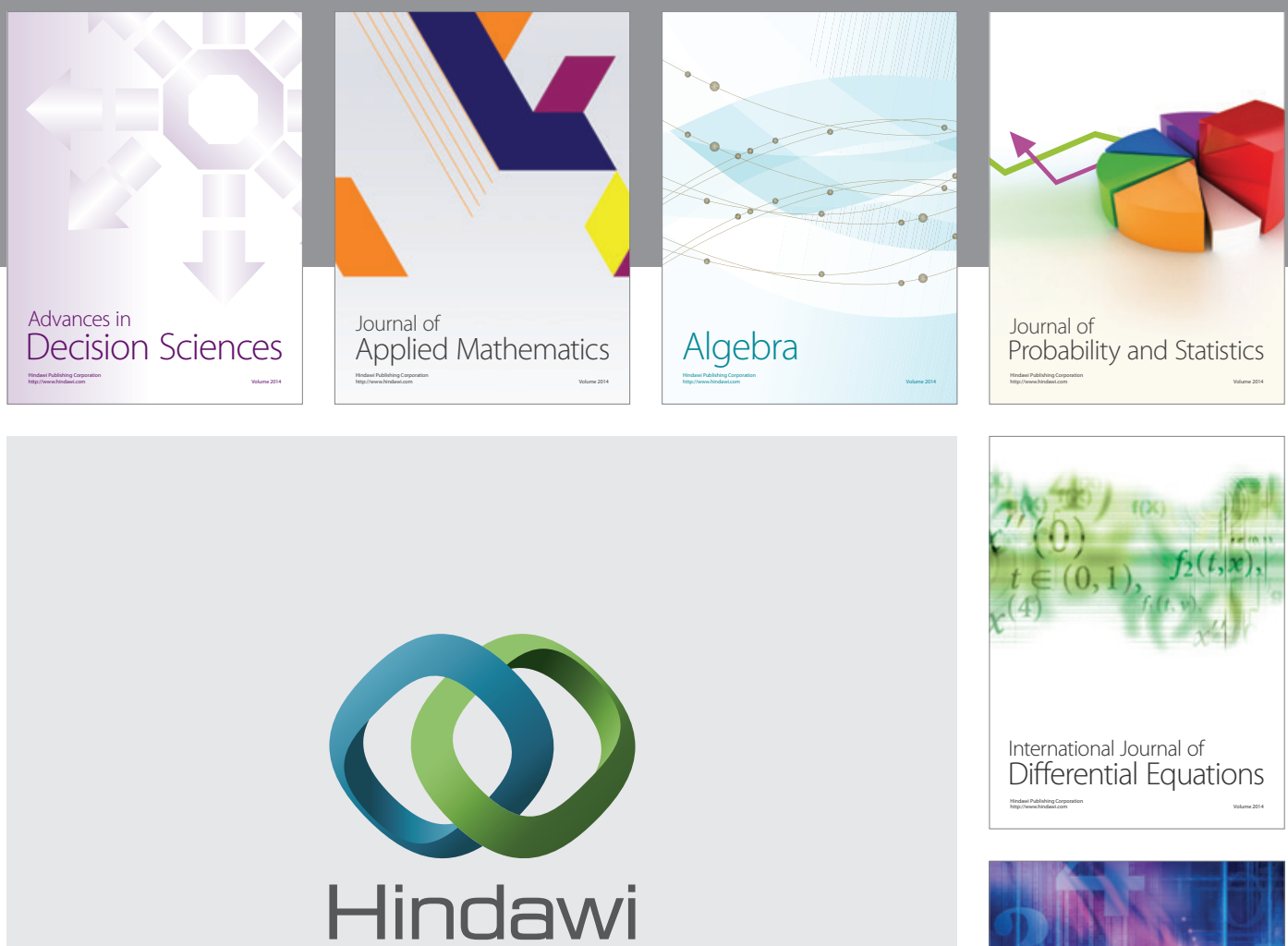

Submit your manuscripts at http://www.hindawi.com
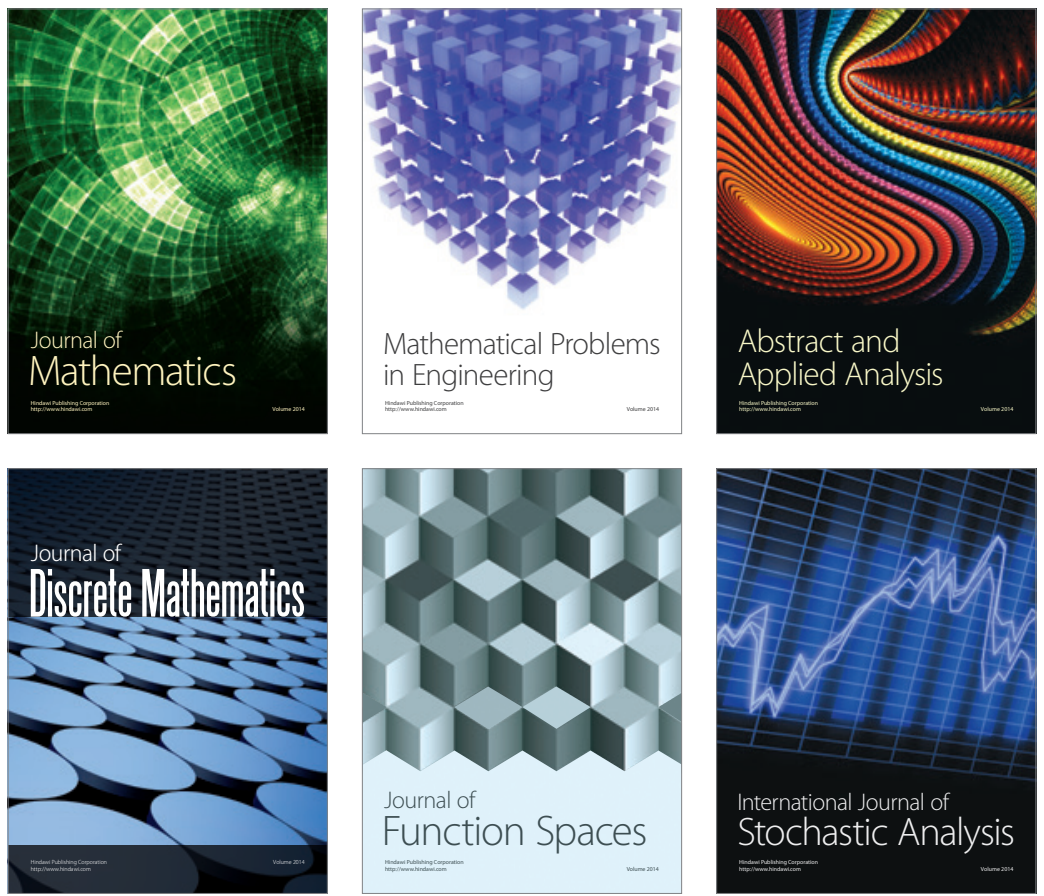

Journal of

Function Spaces

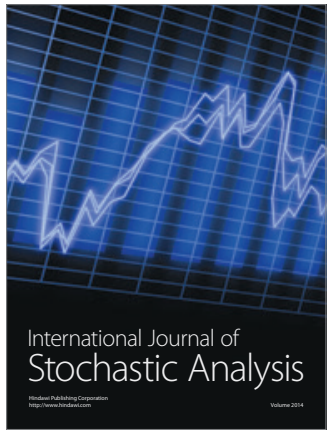

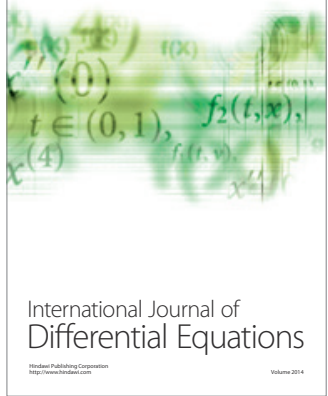
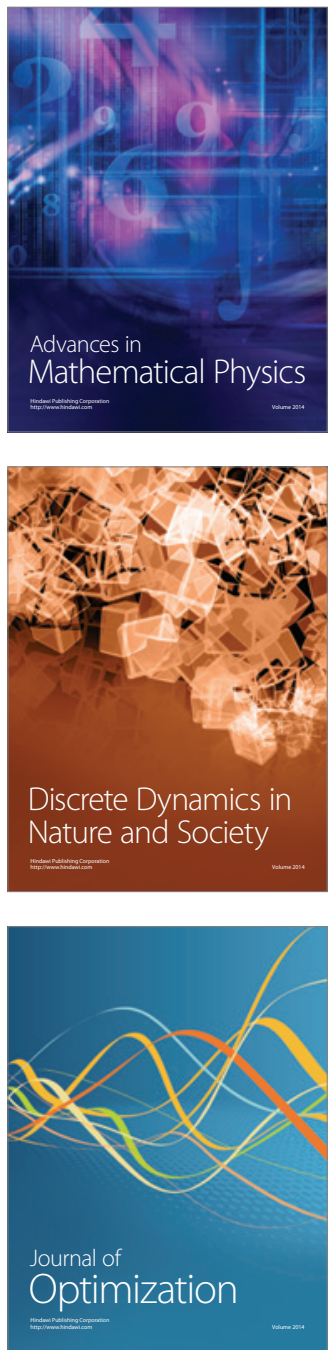